\title{
AN L-BAND POLARIZED ELECTRON PWT PHOTOINJECTOR FOR THE INTERNATIONAL LINEAR COLLIDER (ILC)*
}

\author{
D. Yu, A. Baxter, P. Chen, M. Lundquist, Y. Luo, A. Smirnov \\ DULY Research Incorporated, Rancho Palos Verdes, CA, U.S.A.
}

\section{Abstract}

Plane-wave-transformer (PWT) photoinjectors being developed by DULY Research Inc. are capable of operation in ultra high vacuum and moderate field gradient. Expected performance of a proposed L-band polarized electron PWT injector for the International Linear Collider (ILC) is evaluated in this paper. The projected normalized transverse rms emittance of a $\sim 10 \mathrm{MeV}$ polarized beam is an order of magnitude lower than that calculated for a TESLA pre-accelerator utilizing dc gun and bunch compression.

\section{INTRODUCTION}

A multi-cell, standing-wave, L-band, $\pi$-mode, planewave-transformer (PWT) photoinjector (Fig. 1) with an integrated photocathode in a novel linac structure [1] may be directly applicable as a polarized electron source for the International Linear Collider (ILC). A highly polarized $(>80 \%)$ electron beam is produced from a strained lattice GaAs photocathode illuminated by a circularly-polarized laser $(\lambda \approx 800 \mathrm{~nm})$. The projected normalized transverse rms emittance is about $3 \mathrm{~mm}-\mathrm{mrad}$ (compared with $42.5 \mathrm{~mm}$-mrad in the TESLA preaccelerator design). Thus, the PWT photoinjector may drastically reduce the cost of the complicated damping ring in the ILC. Unlike conventional SLAC-type disk loaded structures, PWT cavities are formed between washers (disks) that are suspended by cooling pipes inside a large cylinder. The "open" cavities are strongly coupled electromagnetically, transforming a TEM-like mode in the annular space (between the cylindrical tank and the disks) into a TM01-like mode on beam axis.

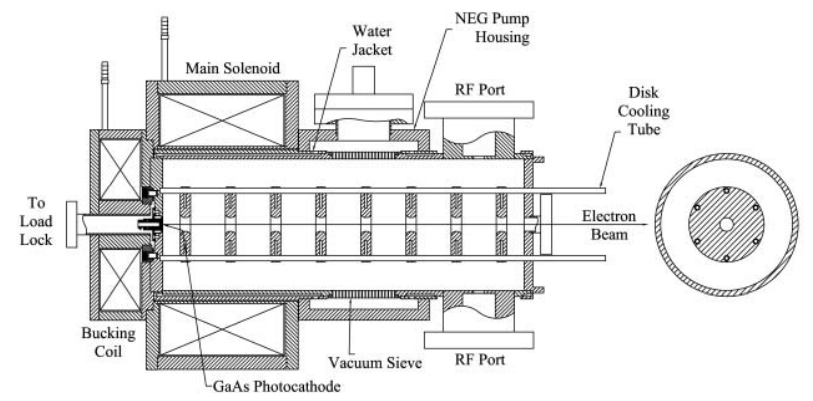

Figure 1: An L-band integrated PWT photoelectron linac. Section view (right) shows 6 disk-support/cooling rods.

The proposed L-band PWT photoinjector has unique features that facilitate meeting the requirements [2] of a polarized electron gun for the ILC.

Cathode - Activation of a GaAs photocathode includes the deposition of cesium onto a perfectly clean GaAs

*Work supported in part by DOE SBIR Grant No. DE-FG0203ER83846. surface to optimize the quantum efficiency (QE) Subsequent reactivation requires that the processing be an integral part of a cathode insertion system in ultra high vacuum. DULY has designed a load-lock system for a previous S-band PWT (Fig. 2c) [3], and this design can be readily scaled to L-band.

Scaling data from the SLAC dc gun with a $20 \mathrm{~mm}$ GaAs photocathode operating at $1.8 \mathrm{MV} / \mathrm{m}$ (giving a peak space charge limit current of 15 A) [4], an L-band PWT operating at $20 \mathrm{MV} / \mathrm{m}$ could have over $500 \mathrm{~A}$ of peak current and still be within the space charge limit. This should meet the high current requirement for the ILC. To generate a polarized electron beam with the required ILC beam time structure within each macropulse, a $\mathrm{Ti}$ Sapphire mode-locked laser producing pulsed, circularly polarized light, such as the scheme suggested by Hovater and Poelker [5], may be used.
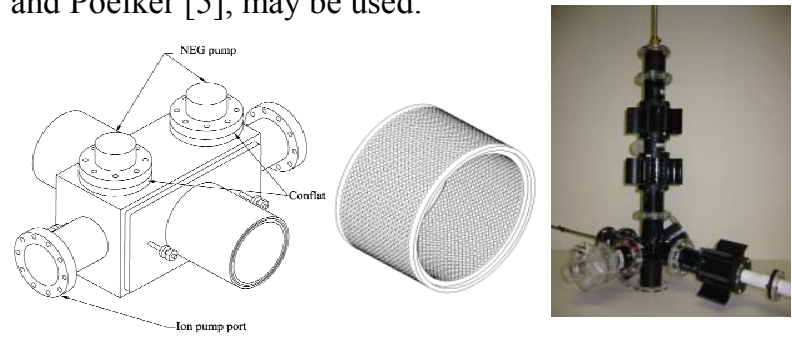

Figure 2: (a) Drawing of a pumping box; and photos of (b) a vacuum-rf sieve, and (c) a load lock model.

Vacuum - An activated GaAs photocathode will be used in ILC's polarized electron injector. The GaAs cathode requires a vacuum of $10^{-11}$ Torr or better in order to sustain an acceptable lifetime and QE. To minimize outgassing, the large PWT tank will be made of $304 \mathrm{~L}$ stainless steel. The separation of the tank wall from the suspended disk assembly allows for a high vacuum conductance that will significantly aid in achieving the UHV requirement for the polarized electron source. Rather than requiring the pumping to occur only through the iris of the disk, the PWT utilizes the large volume between the disks as the primary pumping pathway. Ultra high vacuum is achieved with several non-evaporative getter (NEG) pumps housed in a pumping box (Fig. 2a) that straddles a vacuum-rf sieve (Fig. 2b), which is part of the PWT tank.

High Voltage - To extract the high charge $(3.7 \mathrm{nC}$ per bunch) needed for the ILC electron beam, the cathode must be biased at high voltage. For conventional guns using either dc or pulsed voltage, the rate of decay of the cathode QE increases when the average dark current exceeds $100 \mathrm{nA}$. RF guns rely on the rf fields at the cathode surface to extract the charge. These fields can produce dark current that can destroy the cathode QE [6]. 
This detrimental effect is mitigated by operating the PWT at a low field gradient [7]. For the proposed normalconducting L-band PWT gun it was found that a peak field of $20 \mathrm{MV} / \mathrm{m}$ sufficed. Operating at this relatively low peak field, and with low over-voltage at the irises $(<1.5)$, voltage breakdown is not expected to be a concern with proper $\mathrm{rf}$ conditioning.

Electron Bombardment - A negative electron affinity (NEA) GaAs photocathode is prone to dark current emission when bombarded by electrons or ions. Since electrons have a smaller mass and are more mobile, fieldemitted electrons in the rf cavity can potentially reach the cathode, damaging the activated layer. Recent simulations have shown, however, that the back bombardment of secondary electrons in a PWT is significantly curtailed by operating it at a low field gradient [7], thus helping suppress dark current production. High duty factor dc guns have serious degradation to the cathode $\mathrm{QE}$ due to residual gas and electron impaction; desorbed molecules are ionized by the electron beam and accelerated into the cathode. Being an rf gun, the PWT photoinjector is not expected to suffer as seriously, because of the rapidly reversing electric field.

\section{RF DESIGN OF AN L-BAND PWT PHOTOINJECTOR}

A baseline design of a $7+2 / 2$ cell, L-band PWT is chosen to maximize the field gradient for the available rf power, and to provide sufficient space to accommodate the solenoids, the pumping box and the rf ports. There are several competing design objectives in the PWT. Some of these relate to the electromagnetic performance, and others to vacuum, cooling and dark current considerations. For example, on one hand it is advantageous to have a small aperture on the disks so that the shunt impedance can be maximized for a given rf peak power, in order to provide a sufficiently large E-field needed to capture photoelectrons into the PWT and to improve beam dynamics. However, an aperture that is too small may interfere with the beam and introduce dark current, which can rapidly diminish the life of the cathode. Based on preliminary simulations, an aperture of $15 \mathrm{~mm}$ appears to be a good balance between these competing conditions.

Similarly, a balance must be found for the amplitude of the required peak E-field. Potential sources of secondary electrons are the cathode holder and the PWT irises. To mitigate the secondary electron flow onto the cathode it is necessary to have a low peak E-field. However, a sufficiently large E-field on the cathode surface is required to capture electrons emitted from the cathode into the PWT linac. Based on PARMELA simulations (see Table 1), we choose a peak field of $20 \mathrm{MV} / \mathrm{m}$. This field value results in a low beam emittance while maintaining a reasonable $\mathrm{rf}$ power requirement.

We have considered two options for the rf design of the PWT. The first design option is simple, although at the expense of a lower efficiency. Using two phasesynchronized klystrons coupled to the PWT injector via two symmetrical rf ports provides a total of $20 \mathrm{MW}$ of peak power. This input power will be sufficient to produce the target peak E-field of $20 \mathrm{MV} / \mathrm{m}$ at the cathode. The higher heat load will require 6 cooling rods, and a larger PWT tank. The removal of heat due to dissipated power in the PWT components (disks, rods and tank) is discussed in a companion paper [8]. The 6 rods also help improve the shunt impedance.

Table 1: Comparison of PARMELA simulations for different peak electric field. Initial beam parameters: bunch charge $\mathrm{Q}=3.7 \mathrm{nC}$, beam radius $=5.7 \mathrm{~mm}$, bunch length $=15$ ps. Epeak $=$ peak electric field $(\mathrm{MV} / \mathrm{m}), \mathbf{B}=$ peak magnetic field (Gauss), $\boldsymbol{\varepsilon}=$ normalized rms transverse emittance (mm-mrad), $\mathbf{z}=$ location of emittance minimum, from the exit of the gun $(\mathrm{m}), \boldsymbol{\sigma}=\mathrm{rms}$ beam size $(\mathrm{mm})$, $\mathbf{w}=$ energy gain $(\mathrm{MeV}), \Delta \mathbf{w}=$ rms energy spread $(\%)$.

\begin{tabular}{|c|c|c|c|c|c|c|}
\hline Epeak & $\mathbf{B}$ & $\boldsymbol{\varepsilon}$ & $\mathbf{z}$ & $\boldsymbol{\Sigma}$ & $\mathbf{W}$ & $\Delta \mathbf{w}$ \\
\hline 24 & 725 & 2.0 & 1.8 & 0.7 & 11.9 & 0.03 \\
\hline 22 & 700 & 2.2 & 1.9 & 0.8 & 11.2 & 0.02 \\
\hline 20 & 670 & 3.2 & 1.8 & 0.8 & 10.3 & 0.02 \\
\hline 18 & 638 & 6.8 & 1.4 & 0.7 & 9.4 & 0.05 \\
\hline 16 & 605 & 8.7 & 1.9 & 0.67 & 8.3 & 0.13 \\
\hline 14 & 540 & 18.0 & 2.4 & 1.17 & 6.9 & 0.39 \\
\hline
\end{tabular}

The second option employs only one klystron (10 MW), saving power consumption and klystron cost. In order to maximize the shunt impedance and achieve the target peak field at the photocathode, extensive structural modifications (from our standard PWT design) must be made. Several design variants have been considered, such as adding peripheral rods (Fig. 3a), and introducing the nose of the irises to form re-entrant cavities (Fig. 3b). The peripheral rods do not support the disks, but serve effectively to enhance the Q-factor and the shunt impedance, in the presence of the SS tank (see Table 2).

Table 2: Shunt impedance and peak electric field of several designs of an L-band polarized electron injector.

\begin{tabular}{|c|c|c|c|}
\hline $\begin{array}{c}\text { Design } \\
\text { Variant }\end{array}$ & $\begin{array}{c}\mathbf{r , ~ M \Omega / m} \\
\text { for } \\
\mathbf{8} \text { disks }\end{array}$ & $\begin{array}{c}\text { Epeak, } \\
\text { MV/m, for } \\
\mathbf{1 0} \mathbf{M W} \mathbf{~ r f} \\
\text { power }\end{array}$ & $\begin{array}{c}\text { Epeak, } \\
\mathbf{M V} / \mathbf{m}, \text { for } \\
\mathbf{2 0} \mathbf{M W ~ r f} \\
\text { power }\end{array}$ \\
\hline $\begin{array}{c}\text { Scaled PWT } \\
\text { with 4 rods }\end{array}$ & 11.5 & 11.2 & 15.8 \\
\hline 6 rods & 17.1 & 13.6 & 19.2 \\
\hline 4+12 rods & 23.1 & 15.8 & 22.4 \\
\hline $\begin{array}{c}\text { 4+12rods } \\
\text { +reentrant }\end{array}$ & 31.8 & 18.6 & 26.2 \\
\hline
\end{tabular}

The PWT design configurations listed in Table 2 include options of both a $10 \mathrm{MW}$ and a $20 \mathrm{MW}$ rf input. The "scaled PWT" structure refers to an injector design that is scaled from an earlier DULY S-band PWT to Lband, consisting of disks and rods supported and cooled 
by 4 rods only. The "6 rods" design (Fig. 1) has 2 additional rods, and a larger diameter tank. The " $4+12$ rods" design consists of 4 rods that support/cool the disks, plus 12 peripheral rods in a rod-loaded cavity design (Fig. $3 a)$. The " $4+12$ rods + re-entrant" design has improved $\mathrm{R} / \mathrm{Q}$ due to its re-entrant-like shape as shown in Fig. $3 \mathrm{~b}$. a)

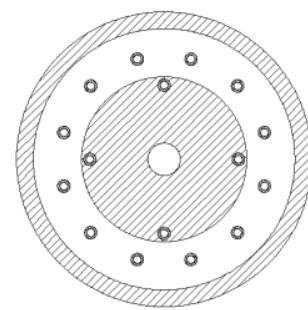

b)

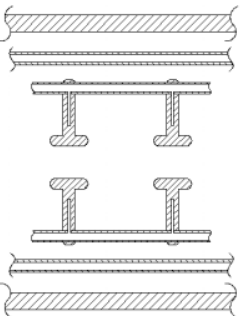

Figure 3: (a) PWT designs with 12 peripheral rods and 4 cooling/supporting rods between disks. (b) Cutaway view showing a re-entrant cavity with an optimized nose on each PWT iris.

Results of preliminary simulations and analysis of an Lband PWT polarized electron photoinjector using the " 6 rod" design are compared with the current TESLA design in Table 3. The beam parameters of the PWT outperform those of the TESLA pre-accelerator design in all categories. The "peripheral-rod" design options will be further investigated as they have better efficiencies than the "6-rod" design, and need only a single klystron to achieve the required peak field.

Table 3: Comparison of TESLA pre-accelerator designs with L-band PWT photoinjector simulations.

\begin{tabular}{|l|c|c|c|}
\cline { 2 - 4 } \multicolumn{1}{l|}{} & $\begin{array}{l}\text { DULY } \\
\text { PWT “6- } \\
\text { Rods” } \\
\text { Design }\end{array}$ & \multicolumn{2}{|c|}{$\begin{array}{l}\text { TESLA 2001 } \\
\text { Technical } \\
\text { Report [2] }\end{array}$} \\
\hline N cells & $7+2 / 2$ & $5+5$ & 17 \\
\hline Length, m & 0.922 & 1.15 & 1.96 \\
\hline Aperture radius a, cm & 1.5 & \multicolumn{2}{|c|}{2.6} \\
\hline $\begin{array}{l}\text { Shunt impedance } \\
|U T|^{2} / P L, M \Omega / m\end{array}$ & 17.1 & 63.8 & 70.8 \\
\hline Acc. gradient, MV/m & 9.6 & 12 & 9.5 \\
\hline Q-factor & 20330 & \multicolumn{2}{|c|}{$\sim 21500$} \\
\hline $\begin{array}{l}\text { Heat (5Hz, 1.37ms, } \\
\text { 10MW/klystron), kW }\end{array}$ & $\begin{array}{c}137 \\
\text { klystrons }\end{array}$ & \multicolumn{2}{|c|}{1 klystron } \\
\hline
\end{tabular}

\section{Beam parameters for $\mathrm{q}=3.7 \mathrm{nC}$ per bunch}

\begin{tabular}{|l|c|c|}
\hline Energy, MeV & 10 & 11.3 \\
\hline Laser pulse length, ns & 0.015 & 2 \\
\hline $\begin{array}{l}\text { Bunch length (rms), } \\
\text { mm }\end{array}$ & 2.0 & 3.4 \\
\hline $\begin{array}{l}\text { Energy spread (rms), } \\
\text { keV }\end{array}$ & 4.0 & 45 \\
\hline $\begin{array}{l}\text { Normalized transverse } \\
\text { emittance (rms), } \\
\text { mm·mrad }\end{array}$ & 3.2 & 42.5 \\
\hline Beam size (rms), mm & 0.8 & 2.6 \\
\hline
\end{tabular}

\section{VACUUM AND THERMAL-HYDRAULIC DESIGN}

The vacuum conductance of the L-band PWT is considerably higher than a scaled S-band version because of the large pumping paths and large holes of the vacuumrf sieve (Fig. 2b). A large pumping box (Fig. 2a) can accommodate up to 8 high-capacity NEG pumps to offset the outgassing of the SS tank and other copper surfaces, resulting in a pressure at the cathode of less than $10^{-11}$ Torr. The vacuum load lock for an L-band PWT can be scaled from the S-band design.

The L-band linear collider uses substantial average rf power. In the worst-case scenario, the L-band PWT design uses two phase-synchronized klystrons, each with a long rf pulse of 1370 microseconds, $10 \mathrm{MW}$ peak power and $5 \mathrm{~Hz}$ rep rate. The heat transferred to the PWT tank wall can be readily removed with a coaxial water jacket and flow diverters inside the cylindrical tank. Heat transferred to the disks and pipes that support them must be removed by water inside the pipes and internal disk channels. To prevent differential thermal expansion among disks (which may detune the rf cavities), they should have the same temperature at steady state. This is accomplished by adjusting the disk channel water flow rate with a variable inlet orifice size for each disk.

Separating the six cooling pipes into three independent cooling circuits allows each circuit to feed water in parallel into sets of 2 or 3 disks. Details of thermalhydraulic analyses are presented in a companion paper [8]. The results show that the cooling capacity of the Lband PWT is adequate.

\section{CONCLUSION}

An L-band photoinjector with the plane-wave-transformer design is proposed as a low-emittance, polarized electron source for the International Linear Collider (ILC). Preliminary rf, vacuum, thermal hydraulic and mechanical considerations demonstrate feasibility of the concept.

\section{REFERENCES}

[1] D. $\mathrm{Yu}$ et al, Proceedings of the SPIN 2004 Conference, Trieste, Italy, October, 2004.

[2] TESLA Technical Design Report, Part II, ed. R. Brinkmann, K. Flottmann, J. Rossbach, P. Schmuser, N. Walker, H. Weise, Chapter 4, http://tesla.desy.de/ new_pages/TDR_CD/PartII/accel.html, March, 2001.

[3] D. Yu et al., Proc. of Particle Accelerator Conference, May 2003, p. 2129.

[4] R. Alley et al., Nucl. Inst. and Meth. A365, 1 (1995).

[5] C. Hovater and M. Poelker, Nucl. Inst. and Meth. A418, 280 (1998).

[6] N. S. Dikansky et al., in Proceedings of the 7th European Particle Accelerator Conference, Vienna, 2000, p.1645.

[7] Y. Luo et al., Proc. of Particle Accelerator Conference, May 2003, p. 2126.

[8] D. Yu et al., these proceedings. 